\title{
Cytomegalovirus: A possible cause of Persistent Refractory Immune Thrombocytopenic Purpura
}

\author{
Raksha Shrestha," Damiano Rondelli, Mingma Thsering Sherpa
}

Department of Hematology-Oncology, College of Medicine, University of Illinois at Chicago, USA

\begin{abstract}
Accepted on
December $5^{\text {th }}, 2013$

DOI Name

http://dx.doi.org/10.3126/jaim.v3i1.10704

Keywords

Cytomegalovirus, Immune Thrombocytopenic

Purpura

Citation

Raksha Shrestha, Damiano Rondelli, Mingma Thsering Sherpa. Cytomegalovirus: A possible cause of Persistent Refractory Immune Thrombocytopenic Purpura. Journal of Advances in Internal Medicine 2014;03(01):42-45.
\end{abstract}

\begin{abstract}
We present a case of a healthy 22 years old who presented with bruising and hematoma following a minor trauma. Investigations showed severe thrombocytopenia but complete hematological investigations did not reveal a cause. An aggressive treatment with steroids, IVIG, anti-D, splenectomy and immunosuppressant proved to be refractory. A subclinical transaminitis prompted an infectious workup which revealed Cytomegalovirus infection. Platelet count improved and remained stable after eradication of Cytomegalovirus. We conclude that infection with CMV should be ruled out in cases of severe refractory immune thrombocytopenic purpura.
\end{abstract}

\section{BACKGROUND}

Idiopathic thrombocytopenia(ITP) is a bleeding disorder characterised by an abnormal decrease in the number of platelets secondary to immune mediated platelet destruction and impaired platelet production. ${ }^{1-4}$ Infections such as Cytomegalovirus, H.Pylori, HIV, and Hepatitis $C$ have been implicated to cause a post-infectious ITP in adults. These aetiologies have been attributed to trigger an autoimmune response against platelets, even though the infections themselves are transient and seemingly neither atypical nor severe in nature. ${ }^{5}$

In the past several years, Cytomegalovirus (CMV) is rising as a prominent aetiology in cases of persistent thrombocytopenia. While treatment of refractory ITP generally involves modalities with potentially harmful risks, eradication of CMV (in positive cases) has shown to help normal treatment regain their effectiveness. Here, we aim to reinforce the need to recognize CMV as an important aetiology in cases of severe persistent thrombocytopenia.

\section{CASE PRESENTATION}

A 22 years old male presented to the emergency department with hematuria and melena. His laboratory findings were dominated by a platelet count of $6 \times 10^{9} /$. A careful medical history revealed that the patient had presented few weeks ago in an outside hospital with multiple bruises on shoulder, arms and legs and a large hematoma on his hand after minor trauma and was found to have platelet count of $1 \times 10^{9} / \mathrm{l}$. He had been diagnosed with ITP based on exclusion of other causes and supported by normal bone marrow biopsy. He had received IVIG, steroids, platelet transfusion and anti-RhD, all of which proved to be refractory. Laparoscopic splenectomy had been performed, but had yielded little response.

\footnotetext{
* Corresponding author

Raksha Shrestha

Clinical Elective Student

Section of Hematology/Oncology,

University of Illinois Hospital and Health Science System and University of Illinois Cancer Center, Chicago, IL, USA

Email: raksha.shres@gmail.com
} 
Initial management of ITP with platelet transfusion and steroids was done. After a few days, patient developed severe hypertension and seizures, which was attributed to high dose steroids. Steroids were tapered and he was started on Levetiracetam, Thromboplastin weekly and Rituximab. MRI revealed progressive reversible leukoencephalopathy, which forced the discontinuation of Rituximab. Patient received platelet transfusions in events of epistaxis and severe thrombocytopenia to which he responded well transiently. During his hospital stay, platelet count remained stable between 10-15 X 10\% /. Patient received platelet transfusion and Nplate (thrombopoiesis-stimulating agent) on the day of discharge. The plan was to receive platelets every third day and Nplate every week.

The patient was noted to have persistently elevated transaminases in his follow up-visit. An infectious workup revealed an acute CMV infection. He was admitted with a platelet count of 11000 and was started on intravenous Foscarnet but later switched to oral Valganciclovir due to deep venous thrombosis, a complication that developed after PICC line insertion. $\mathrm{N}$-plate was discontinued; platelet transfusion and anticoagulants were held due to fear of exacerbation of clot. Platelet counts were similar as at the time of admission while being treated for CMV viremia. He was discharged after a week of hospital stay.

\section{Outcome and Follow up care}

In the follow up visits, CMV was detected but not quantifiable and his platelet count was found to be stable and improving.

\section{Past medical history}

The patient had a remote history of Hodgkin's disease diagnosed and treated at an outside hospital at 14 years of age. He had been assured that there was no evidence of recurrence 2 years ago and had not followed up since.

\section{DISCUSSION}

Immune Thrombocytopenic Purpura is an acute, self-limited disease in the vast majority of children whereas in adults, ITP has an insidious onset, often with a chronic course. The incidence of ITP in adults increases with age and most cases achieve stable remission. ${ }^{6,7,8}$. Common presentations of ITP are petechiae and purpura but can include fatal complications such as intracerebral and intracranial bleeding. Prognosis for most patients is generally good with a small percentage of mortality, attributed mainly to severe refractory thrombocytopenia. ${ }^{9}$

ITP is a cause of primary immune thrombocytopenia whereas secondary causes are HIV, Hepatitis C, SLE, hematological malignancies and drug-induced. ITP is often a diagnosis of exclusion as other causes are more common and readily reversible. ${ }^{10}$

Isolated thrombocytopenia on blood films with rest of the complete blood count entirely normal and absence of any other non-immune or secondary immune causes prompts one to make a diagnosis of ITP. Standard practice is to commence treatment with steroid with or without immunoglobulin when platelet count falls below $20 \times 10^{9} / \mathrm{l}$ or $50 \times 10^{9} / \mathrm{I}$ in the presence of bleeding. ${ }^{11}$ Splenectomy has been the next most effective treatment for ITP with the highest rate of complete and durable remissions. ${ }^{12}$ An anit-CD-20 monoclonal antibody, Rituximab has been associated with an improvement in platelet count response in $60 \%$ of patients.

ITP is considered refractory when it presists for more than three months with a platelet count $<30 \times 10^{9} / /$ and does not respond to splenectomy and rituximab. ${ }^{13}$ In cases of postsplenectomy refractory thrombocytopenia, immunosuppressants (cyclophoshamide, azathioprine, cyclosporine, mycophenolatemefotil), vincaalkaloids(vinblastine, vincristine), thrombopoiesis-stimulating agents and androgenic treatment(Danazol) have been used but not always responsive.

In the past several years, cases of apparently idiopathic ITP have been found to be secondary to an unsuspected, persistent infection. Among other infectious agents, Cytomegalovirus is gaining recognition as an uncommon etiology. CMV usually manifests in an immunocompromised host but is usually asymptomatic and usually detected retrospectively in the immunocompetent. It is a well recognized cause of 'glandular fever'- like illness with atypical mononuclear cells in peripheral smear. Sites most often involved include the lung, liver, spleen, gastrointestinal tract and CNS. ${ }^{14}$ In our case, the presence of persistent subclinical transaminitis prompted an acute infection workup. CMV has been reported to both cause and perpetuate ITP in pediatric as well as adult population. ${ }^{5,15,16}$

Several hypotheses have been put forward regarding attribution of virus and bacteria to ITP. While molecular mimicry and chronic infection leading to immune dysregulation are possible, CMV is able to directly infect megakaryocytes and thereby decrease platelet production. ${ }^{17,18,19}$ This seems to be the best explanation for the failure to respond to any of the conventional ITP therapies, especially as their primary effects are to block platelet destruction, not to stimulate platelet production. ${ }^{20}$ Cytomegalovirus has been detected via culture, serologies, antigen assays, PCR, and cytopathology. 
Treatment options invariably consist of antivirals, which remained the same for our case. In a study, a combination of both IV ganciclovir and IV cytogam had been used to optimize rapid suppression of the $\mathrm{CMV} .{ }^{5}$ The same study showed that use of steroids and other immunosuppressive treatment may exacerbate the primary CMV infection and worsen the ITP, or at least prevent it from improving. Also of interest is that, when CMV is controlled by anti-viral therapies, normal treatments for ITP, such as IVIG and splenectomy regain their effectiveness. ${ }^{5}$ The response rate of platelet counts following

\section{REFERENCES}

1. Bussel JB, Kuter DJ, George JN et al. AMG 531, a thrombopoiesis-stimulating protein, for chronic ITP. NEJM 2006; 355(16): 1672-1681. http://dx.doi.org/10.1056/NEJMoa054626

2. Ballem PJ, Segal GM, Stratton JR, Gemsheimer T, Adamson JW, Slichter SJ. Mechanisms of thrombocytopenia in chronic autoimmune thrombocytopenic purpura. Evidence of both impaired platelet production and increased platelet clearance. J Clin Invest 1987;80:33-40. http://dx.doi.org/10.1172/JCl113060

3. Isaka Y, Kambayashi J, Kimura K et al. Platelet production, clearance and distribution in patients with idiopathic thrombocytopenic purpura. Thromb Res 1990;60(2):121-131. http://dx.doi.org/10.1016/0049-3848(90)90291-J

4. Louwes H, Zeinali Lathori OA, Vellenga E, de Wolf JT. Platelet kinetic studies in patients with idiopathic thrombocytopenic purpura. Am J Med 1999;106(4): 430-4. http://dx.doi.org/10.1016/S0002-9343(99)00054-6

5. Dimaggio D, Anderson A and Bussel JB. Cytomegalovirus can make immune thrombocytopenic purpura refractory. Br J Hematol 2009; 146(1): 104-12. http://dx.doi.org/10.1111/j.1365-2141.2009.07714.x

6. Frederiksen $\mathrm{H}$, Schmidt $\mathrm{K}$. The incidence of idiopathic thrombocytopenic purpura in adults increases with age. Blood. 1999;94: 909-913.

7. Stevens W, Koene H, Zwaginga JJ, Vreugdenhil G. Chronic idiopathic thrombocytopenic purpura: present strategy, guidelines and new insights. Neth J Med 2006; 64(10): 356-63.

8. McMillan R, Durette C. Long-term outcomes in adults with chronic ITP after splenectomy failure. Blood. 2004; 104(4): 956-60. treatment of CMV has seen to vary widely between studies.

The presence of isolated thrombocytopenia with or without history of fever and myalgia, presence of subclinical transaminitis and atypical lymphocytes on smear should incline a clinician to suspect CMV, especially in endemic areas. These considerations can avoid the aggressive treatments of refractory ITP and their fatal side effects. Nevertheless, a limited or no response to ITP specific therapies such as IVIG, anti-D, steroids, immunosuppressants should prompt hematologists to test CMV as a part of their ITP investigation.

http://dx.doi.org/10.1182/blood-2003-11-3908

9. Arnold DM, Kelton JG. Current options for the treatment of idiopathic thrombocytopenic purpura. Semin Hematol 2007;44(4 Suppl 5):S12-23 http://dx.doi.org/10.1053/j.seminhematol.2007.11.003

10. Altemimi H, Brown J, Low KE, Al-Juboori S, Coates P. H. Pylori-induced thrombocytopenia in a Caucasian female. BMJ Case Reports 2010; 10: 1136.

11. George JN, Woolf SH, Rashkob GE, et al. Idiopathic thrombocytopenic purpura: a practice guideline developed by explicit methods for the American Society of Hematology. Blood 1996;88:3-40.

12. Kjouri K, Vesely SK, Terrell DR, George JN. Splenectomy for adult patients with idiopathic thrombocytopenic purpura: a systematic review to assess long-term platelet count responses, prediction of response, and surgical complications. Blood 2004; 104:2623. http://dx.doi.org/10.1182/blood-2004-03-1168

13. George JN. Management of patients with refractory immune thrombocytopenic purpura. J ThrombHaemost 2006; 4: 1664. http://dx.doi.org/10.1111/j.1538-7836.2006.02013.x

14. Cunha BA. Cytomegalovirus pneumonia: communityacquired pneumonia in immunocompetent hosts. Infect Dis Clin North Am. 2010;24(1):147-58. http://dx.doi.org/10.1016/j.idc.2009.10.008

15. Sawanobori M, Nakagawa Y, Inoue Y, Sujuki K. Severe thrombocytopenia after cytomegalovirus infection in an immunocompetent host-correlation between MCV infection and platelet count in an immunocompetent host.Nihon Rinsho Meneki Gakkai Kaishi 1997;20(2):134-8.

http://dx.doi.org/10.2177/jsci.20.134

16. Gural A, Gillis S, Gafanovich A et al. Massive intracranial bleeding requiring emergency splenectomy in a patient 
with CMV-associated thrombocytopenia. Haemostasis 1998; 28(5): 250-5.

17. Xiao $Y$, Lin $W$, Liu $Q$, Jin $R$ and Fei H. Direct Infection of colony forming unit-megakaryocyte by human cytomegalovirus contributes the pathogenesis of idiopathic thrombocytopenic purpura. Journal of Huazhong University of Science and Technology 2006; 26: 555-557. http://dx.doi.org/10.1007/s11596-006-0518-3

18. Mizutani K, Azuma E, Komada Y, et al. An infantile case of cytomegalovirus induced idiopathic thrombocytopenic purpura with predominant proliferation of CD10 positive lymphoblast in bone marrow. ActaPediatrica Japonica 37, 71-74.

19. Crapnell K, Zanjani E, Chaudhuri A, et al. In vito infection of cytomegalovirus infection of megakaryocytes and their precursors by human cytomegalovirus. Blood 2000; 95; 487-492.

20. Psaila SK, Bussel JB. Refractory immune thrombocytopenic purpura: current strategies for investigation and management. British Journal of Haematology 2008; 2143: 16-26. http://dx.doi.org/10.1111/j.1365-2141.2008.07275.x 